\title{
Motivación de los estudiantes hacia el uso de la tecnología para el aprendizaje de las matemáticas.
}

\section{Motivation of students towards the use of technology for learning of mathematics.}

\section{Motivación hacia el uso de la tecnología para el aprendizaje}

\author{
Raquel Vera Velázquez, MSc ${ }^{(1)}$ \\ Kirenia Maldonado Zúñiga, MSc. (2) \\ Wilfrido Javier Del Valle Holguín, Dr ${ }^{(3)}$ \\ Pedro Valdés Tamayo. $\mathrm{PhD}^{(4)}$ \\ (1) Universidad Estatal del Sur de Manabí, Jipijapa, Ecuador. email: raquelita2015vera@gmail.com \\ (2) Universidad Estatal del Sur de Manabí, Jipijapa, Ecuador. email: kmzmaldonado@ gmail.com \\ (3) Universidad Estatal del Sur de Manabí, Jipijapa, Ecuador. email: wilfridojdelvalle@yahoo.es \\ (4) Universidad Estatal del Sur de Manabí, Jipijapa, Ecuador. email: pvaldestamayo@ gmail.co
}

Contacto:raquelita2015vera@gmail.com.

Recibido: 18-02-2020 Aprobado: 14-03-2020

\section{Resumen}

Este trabajo nos describe la construcción y validación de un cuestionario de estudio de la motivación hacia las matemáticas y su aprendizaje con el uso de la tecnología. Se realiza un análisis componencial a partir de su aplicación a 229 estudiantes de ingeniería Agropecuaria, en Sistemas Computacionales y Computación y Redes de la Universidad Estatal del Sur de Manabí. Se aplicó una encuesta para evaluar cuatro aspectos diferentes de la motivación de los estudiantes hacia el uso de la tecnología en el proceso de enseñanza y aprendizaje de las matemáticas, que consideramos importantes: utilidad de la tecnología en las matemáticas, motivación de los estudiantes por la implementación de la tecnología en las matemáticas, no utilidad de la tecnología en las matemáticas, y resultados de los estudiantes con respecto al aprendizaje de las matemáticas con el uso de la tecnología. El análisis factorial del cuestionario da como resultado un modelo de cinco factores como el más representativo, con un factor residual y cuatro factores relevantes. Este análisis confirma la congruencia del cuestionario con el planteamiento inicial y prueba su fiabilidad y consistencia interna. El análisis de consistencia interna por factor nos permitió identificar que los primeros cuatro factores del modelo encontrado para nuestro estudio son relevantes. El último puede considerarse como un factor residual, ya que tiene una baja consistencia interna. De manera general, los cuatro factores relevantes que han surgido del análisis factorial muestran la congruencia del cuestionario con la estructura establecida, que trataba de evaluar los cuatro factores iniciales.

Palabras clave: Actitudes, análisis factorial, estudiantes universitarios, matemáticas, tecnología.

\section{Summary}

This work describes the construction and validation of a questionnaire to study the motivation towards mathematics and its learning with the use of technology. A component analysis is carried out from its application to 229 students of Agricultural Engineering, in Computer Systems and Computation and Networks of the State University of the South of Manabí. A survey was applied to evaluate four different aspects of students' motivation towards the use of technology in the process of teaching and learning of mathematics, which we consider important: usefulness of technology in mathematics, motivation of students for Implementation of technology in mathematics, non-usefulness of technology in mathematics, and student results regarding the learning of mathematics with the use of technology. The factorial analysis of the questionnaire results in a five-factor model as the most representative, with a residual factor and four relevant factors. This analysis confirms the 
congruence of the questionnaire with the initial approach and proves its reliability and internal consistency. The internal consistency analysis by factor allowed us to identify that the first four factors of the model found for our study are relevant. The latter can be considered as a residual factor, since it has a low internal consistency. In general, the four relevant factors that have emerged from the factor analysis show the congruence of the questionnaire with the established structure, which sought to evaluate the four initial factors.

Keywords: Attitudes, factor analysis, university students, mathematics, technology

\section{Introducción}

La evaluación de la motivación de los estudiantes hacia una disciplina, un contenido específico, una metodología o cualquier herramienta didáctica, es un tema de interés tanto para la investigación científica como para la práctica educativa, por su influencia en el proceso enseñanza-aprendizaje así como por el efecto de la educación sobre las mismas. Una de las áreas del conocimiento dentro de la que se han analizado de forma más sistemática es la motivación hacia las Matemáticas (Gil, Blanco y Guerrero, 2005). No obstante, sigue siendo un desafío el detectar la influencia de las actitudes y los factores emotivos en el aprendizaje de las matemáticas. Este desafío se incrementa cuando se añaden factores adicionales, como incorporar la tecnología en las aulas (Cretchley y Harman, 2001).

El estudio de las actitudes de los estudiantes ante el proceso de enseñanza y aprendizaje de las matemáticas con tecnología es un tema que ha despertado el interés de diversos investigadores en
Educación Matemática desde diferentes perspectivas (Cretchley, 2007; Cretchley y Harman, 2001; Fogarty, Cretchley, Ellerton y Konki, 2001; Galbraith y Haines, 1998;

Gómez-Chacón y Haines, 2008; Pierce, Stacey y Barkatsas, 2007). Algunas de esas investigaciones reportan que al trabajar temas de matemáticas con el apoyo de la tecnología, aumenta notablemente la motivación de los estudiantes hacia el aprendizaje de las matemáticas, registrándose un cambio positivo en las actitudes hacia esta materia (Ursini, Sánchez y Orendain, 2004).

El objetivo de este trabajo es el diseño y validación de un cuestionario para medir la motivación de los estudiantes con respecto al empleo de la tecnología en el proceso de enseñanza y aprendizaje de las matemáticas. Nuestro interés en esta investigación surge de reconocer la influencia de la motivación e interés en la implementación de experiencias de enseñanza y aprendizaje de las Matemáticas que impliquen el uso de nuevas tecnologías.

Los resultados que obtengamos al aplicar el cuestionario servirán como información preliminar en el diseño de experiencias docentes que hagan uso de la tecnología en la enseñanza de las matemáticas.

\section{Materiales y Métodos}

Los participantes fueron estudiantes del primero y segundo semestres de Ingeniería Agropecuaria, Computación y Redes y Sistemas Computacionales de la Universidad Estatal del Sur de Manabí. Un total de 229 estudiantes respondieron el cuestionario. (tabla 1)

\begin{tabular}{|l|l|l|l|l|l|}
\hline Carrera & Grupos & Especialidad & Femenino & Masculino & $\begin{array}{l}\text { Total de } \\
\text { Alumnos }\end{array}$ \\
\hline Agropecuaria & 5 & Ingeniería & 50 & 39 & 89 \\
\hline Computación y Redes & 3 & Ingeniería & 45 & 35 & 80 \\
\hline $\begin{array}{l}\text { Sistemas } \\
\text { Computacionales }\end{array}$ & 2 & Ingeniería & 40 & 15 & 60 \\
\hline
\end{tabular}

Tabla 1. Distribución de la muestra

Se elaboró un cuestionario para evaluar cuatro aspectos diferentes de la motivación de los estudiantes hacia el uso de la tecnología en el proceso de enseñanza y aprendizaje de las matemáticas, que consideramos importantes:

1- Utilidad de la tecnología en las matemáticas.

2- Motivación de los estudiantes por la implementación de la tecnología en las clases de matemáticas
3- No utilidad de la tecnología en las matemáticas.

4- Resultado de los estudiantes con respecto al aprendizaje de las matemáticas con el uso de la tecnología.

\section{Distribución de la muestra.}

El cuestionario consta de 35 parámetros y para su construcción se analizaron escalas previamente 
validadas en diferentes investigaciones, encontrando que los diferentes aspectos que pretendemos evaluar se cubren con los aspectos pertenecientes a las sub escalas correspondientes a: la interacción de las matemáticas con el ordenador, la experiencia del uso de la tecnología en el aprendizaje de las matemáticas y la motivación por la integración de la tecnología en las matemáticas. Los estudiantes debían indicar su grado de acuerdo o desacuerdo con las afirmaciones propuestas por medio de una escala de Likert de 5 valores.

La interpretación de los factores (tabla 2), la hemos realizado en función de los ítems que más aportan a la saturación del componente, es decir, los ítems con mayor comunalidad.En la tabla anterior se hace referencia a la varianza por factor, teniendo en cuenta el modelo de 5 factores aplicado.

\begin{tabular}{|l|l|l|}
\hline Factores & Frecuencia & Varianza \\
\hline I & 11 & 30 \\
\hline II & 12 & 6.75 \\
\hline III & 4 & 5.15 \\
\hline IV & 4 & 4.49 \\
\hline V & 4 & 4.13 \\
\hline Total & 35 & \\
\hline
\end{tabular}

Tabla \#2: Interpretación de factores relevantes del modelo de 5 factores.

El gráfico 1 muestra la frecuencia por factor donde podemos observar el comportamiento de los factores del grupo I y II fueron los de más aceptación por los estudiantes en la investigación. y el grupo III, IV y $\mathrm{V}$ en menor escala.

Grafico \# 1:Frecuencia por factor.

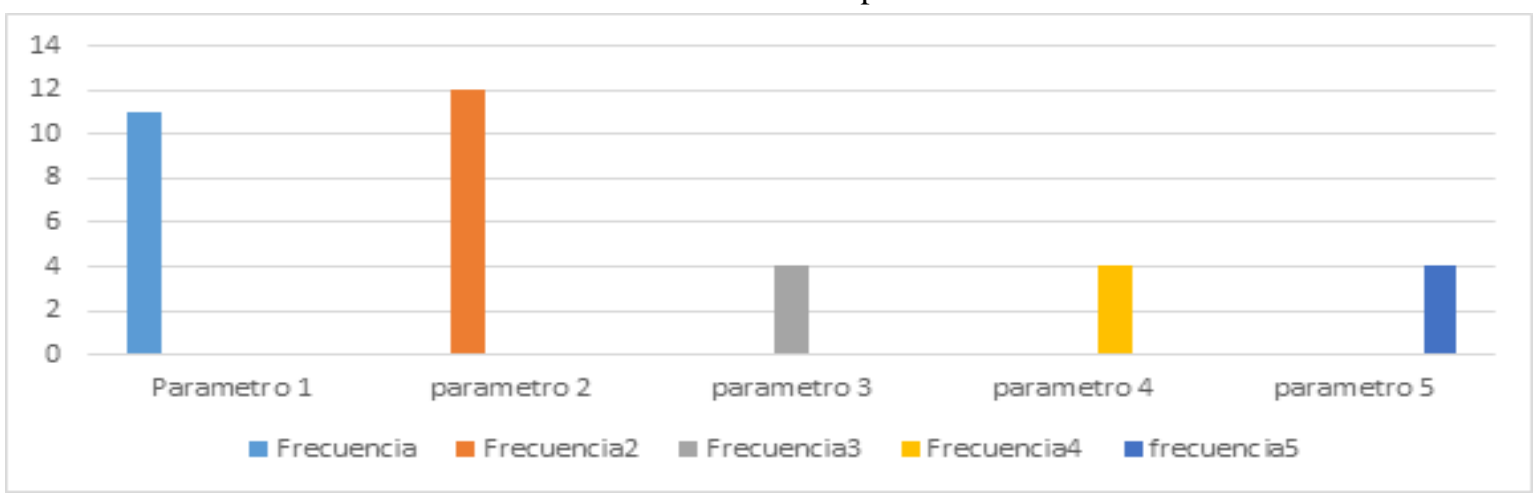

El gráfico 2 describe la varianza por grupo de factores donde el grupo I y II reflejan el interés de los estudiantes y les motiva el uso de la tecnología en las clases de matemáticas no así en los grupo III Y IV, lo que representa que el $30 \%$ de la varianza nos da la consistencia interna del modelo de los cinco factores y que los resultados del modelo es válido para la motivación de los estudiantes por el uso de la tecnología en las clases de Matemática.

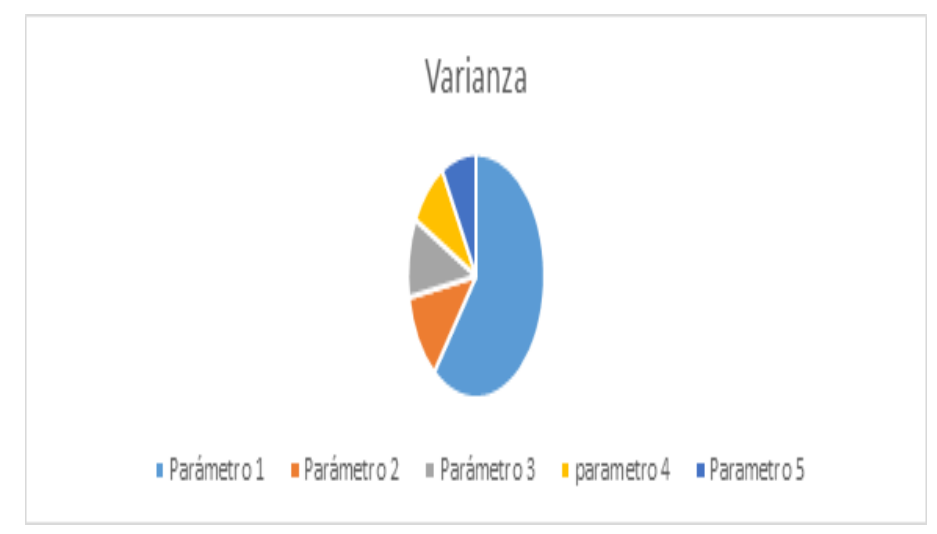

Gráfico \# 2. Varianza por factor. 
Para medir el grado de interacción del pensamiento matemático de los estudiantes con el ordenador involucrando la utilidad del uso de la tecnología en hacer y aprender matemáticas, y resultados del modelo es válido para la motivación e interés de los estudiantes acerca de su aprendizaje de las matemáticas con tecnología consideramos los ocho parámetros (tabla 3) de la sub escala "Interacción con el ordenador y las matemáticas" de Galbraith y Haines (1998, 2000).
Para evaluar la motivación de los estudiantes hacia el uso de la tecnología en las matemáticas se tomaron doce parámetros doce parámetros de Cretchley y Harman (2001); Cretchley (2007); Fogarty et al. (2001) de Pierce, Stacey y Barkatsas (2007) consideramos recogidos en la (tabla 4).

\begin{tabular}{|c|c|}
\hline 1 & $\begin{array}{l}\text { Las computadoras me ayudan a aprender las matemáticas con mayor facilidad proporcionándome } \\
\text { ejemplos de manera interactiva. }\end{array}$ \\
\hline 2 & Me resulta difícil interpretar las ideas de lo mostrado en la pantalla a mi mente. \\
\hline 3 & $\begin{array}{l}\text { La facilidad de los cálculos aritméticos, hace que me concentre mejor en las operaciones } \\
\text { esenciales de las matemáticas. }\end{array}$ \\
\hline 4 & Al leer la pantalla del ordenador, me distraigo y paso por alto las ideas matemáticas. \\
\hline 5 & $\begin{array}{l}\text { Considero que el texto en el ordenador y la copia impresa en un papel son importantes para tomar } \\
\text { notas. }\end{array}$ \\
\hline 6 & $\begin{array}{l}\text { Cuando termino una sesión de actividades en la computadora, casi nunca reviso el material } \\
\text { realizado. }\end{array}$ \\
\hline 7 & El seguimiento a la hoja de trabajo pone mi atención fuera de las operaciones matemáticas. \\
\hline 8 & $\begin{array}{l}\text { El ordenador me ayuda a relacionar los conocimientos, como por ejemplo la forma de los gráficos, } \\
\text { y sus ecuaciones. }\end{array}$ \\
\hline 9 & El uso de la tecnología para cálculos me facilita hacer las aplicaciones más realistas. \\
\hline 10 & Las computadoras están cambiando el modo de hacer las matemáticas. \\
\hline 11 & Los símbolos y el lenguaje Matemático son bastante complicados sin el uso de la tecnología. \\
\hline
\end{tabular}

Tabla 3. Parámetros del 1- 11 "Interacción con el ordenador y las matemáticas”

\begin{tabular}{|l|l|}
\hline 12 & Me motivo a usar el ordenador para resolver operaciones Matemáticas. \\
\hline 13 & Es importante utilizar las computadoras para el aprendizaje de las Matemáticas. \\
\hline 14 & Resolver operaciones Matemáticas con el ordenador se hace más interesante. \\
\hline 15 & El uso de las computadoras me ayuda aprender las Matemáticas. \\
\hline 17 & El poder del ordenador hace más fácil explorar ideas Matemáticas. \\
\hline 18 & Me gusta explorar métodos Matemáticos e ideas utilizando la tecnología. \\
\hline 20 & Disponer de la tecnología me permite probar diferentes métodos y enfoques. \\
\hline 21 & Me gusta aprender las Matemáticas con la ayuda de la computadora. \\
\hline 22 & El uso de software hace el aprendizaje de las Matemáticas más interesante. \\
\hline 23 & $\begin{array}{l}\text { Por propia elección usaré las veces que sea necesario software para el aprendizaje de las } \\
\text { Matemáticas. }\end{array}$ \\
\hline
\end{tabular}

Tabla 4. Parámetros del 12-23 "Motivación de los estudiantes hacia el uso de la tecnología en las clases de Matemáticas".

Para medir la motivación al uso de la tecnología cuando se hace y se aprende matemáticas, así como para evaluar el interés de los estudiantes hacia el uso de la tecnología en su aprendizaje de las matemáticas. Considerando estos parámetros como la no utilidad de la tecnología en las matemáticas evaluados en la Tabla 5 del 24 al 27. 


\begin{tabular}{|c|c|}
\hline 24 & $\begin{array}{l}\text { Sé que las computadoras son importantes pero no siento la necesidad de usarlas para aprender } \\
\text { las Matemáticas. }\end{array}$ \\
\hline 25 & Las computadoras son buenas herramientas para los cálculos pero no para el aprendizaje. \\
\hline 26 & $\begin{array}{l}\text { Pienso que el uso de la tecnología es nuevo y extraño para que valga la pena en el aprendizaje } \\
\text { de las Matemáticas. }\end{array}$ \\
\hline 27 & $\begin{array}{l}\text { Pienso que el uso de la tecnología es una pérdida de tiempo en el aprendizaje de las } \\
\text { Matemáticas. }\end{array}$ \\
\hline
\end{tabular}

Tabla 5. Parámetros del 24 - 27 "No utilidad de la tecnología en las Matemáticas”.

Para evaluar la motivación de los estudiantes con respecto a su experiencia personal del uso de software en su aprendizaje de las matemáticas seleccionamos cuatro parámetros (tabla 7) de Cretchley y Harman (2001) y por último, para medir el gusto por la integración de la tecnología en las matemáticas seleccionamos cuatro ítems tomados de Nguyen y Kulm (2005) de un total de 16 parámetros de la escala general de estos autores, considerando solamente aquellos que involucran los términos ordenador y matemáticas (tabla 6).

\begin{tabular}{|l|l|}
\hline 28 & He encontrado software muy útil para el aprendizaje de las Matemáticas. \\
\hline 29 & Aprender a hacer software para hacer Matemáticas es frustrante. \\
\hline 30 & En términos generales vale la pena aprender a utilizar software para hacer las Matemáticas. \\
\hline 31 & Las tareas por computadora son claras y fáciles de leer. \\
\hline
\end{tabular}

Tabla 6. 28-31 "Experiencia de Matemáticas con tecnología"

Por último, para medir el gusto por la integración de la tecnología en las matemáticas seleccionamos cuatro parámetros de la escala general, considerando solamente aquellos que involucran los términos ordenador y matemáticas, mostrando buena aceptación por los estudiantes en estos parámetros del modelo. (tabla 7).

\begin{tabular}{|l|l|}
\hline 32 & $\begin{array}{l}\text { La retroalimentación inmediata de la computadora es útil para resolver problemas de } \\
\text { Matemáticas. }\end{array}$ \\
\hline 33 & $\begin{array}{l}\text { La revisión de la lección en la tarea por computadora me ayuda a repasar los conceptos } \\
\text { Matemáticos. }\end{array}$ \\
\hline 34 & $\begin{array}{l}\text { Los exámenes de Matemática por computadora con puntuación inmediata me ayudan a evaluar } \\
\text { mi propio entendimiento y rendimiento. }\end{array}$ \\
\hline 35 & La geometría es más clara de entender con el uso de la tecnología. \\
\hline
\end{tabular}

Tabla 7. 32-35 "Matemáticas y tecnología"

El administrador del cuestionario realizó la encuesta en el aula, el propio investigador por grupos de estudiantes, durante una sesión de clase. Antes de entregar el cuestionario, el evaluador proporcionó las instrucciones del llenado y la finalidad de la aplicación del cuestionario. No se limitó el tiempo de respuesta; sin embargo, todos los estudiantes dedicaron menos de 20 minutos a completar el cuestionario.

\section{Resultados}

El primer análisis que hemos realizado ha sido detectar la confiabilidad del test construido. Para determinar el grado de homogeneidad existente entre los elementos de la escala en conjunto, se calculó el Coeficiente Alfa de Cronbach para el total de los parámetros, resultando ser de 0.73 , y de 0.77 basado en elementos estandarizados. Por lo tanto se considera, que la escala tiene una buena consistencia interna.

En segundo aspecto hemos analizado la forma en se agrupan los parámetros de la encuesta aplicada y para ello se realizó un análisis factorial exploratorio.

El objetivo fue determinar factores comunes de los parámetros formando categorías adecuadas que pudieran reducir de manera general los diferentes aspectos de la motivación que queremos medir: El análisis factorial se llevó a cabo por el método de componentes obteniendo ocho factores con valor mayor que uno, explicando el 60,51\% de la varianza total. 
El determinante de la matriz de correlaciones (1.15E007) indica que el grado de inter-correlación entre las variables es alto; condición inicial que debe cumplir este tipo de análisis. Los índices de Kaiser-MeyerOlkin de adecuación de la muestra $(\mathrm{KMO}=0.905)$ y el contraste de esfericidad de Bartlett $(B=3362.8$; $\mathrm{p}<0.001)$ indican una muy buena adecuación de los datos para el tipo de análisis factorial seleccionado.

Para obtener una estructura más simple se realizó una rotación Varimax con normalización de Kaiser. Se procedió a limitar los factores, obteniendo que la solución más interpretable era un modelo de cinco factores.

Por último, hemos calculado los Coeficientes Alfa de Cronbach para cada componente asociada cada factor. (tabla.8)

El análisis de consistencia interna por factor nos permitió identificar que los primeros cuatro factores del modelo encontrado para nuestro estudio son relevantes. El último puede considerarse como un factor residual, ya que tiene una baja consistencia interna.

La interpretación de los factores (tabla 9), la hemos realizado en función de los parámetros que más aportan a la saturación del componente, es decir, los parámetros con mayores factores comunes.

La categorización del primer factor recayó básicamente en elementos tales como los ítems 9, 10, y 11 , los cuáles se refieren a que la tecnología es útil para hacer y aprender Matemáticas. El parámetro 8 podría considerarse como el menos homogéneo en este factor.

\begin{tabular}{|c|c|c|c|}
\hline Factor & $\begin{array}{c}\text { Nro de } \\
\text { parámetros }\end{array}$ & $\begin{array}{c}\% \\
\text { Varianza }\end{array}$ & $\begin{array}{c}\text { Coeficiente } \\
\text { alfa }\end{array}$ \\
\hline I & 11 & 30.00 & $0.873 / 0.875^{*}$ \\
\hline II & 12 & 6.75 & $0.847 / 0.860^{*}$ \\
\hline III & 4 & 5.15 & $0.705 / 0.709^{*}$ \\
\hline IV & 4 & 4.49 & $0.627 / 0.626^{*}$ \\
\hline V & 4 & 4.13 & $0.508 / 0.510^{*}$ \\
\hline
\end{tabular}

*Coeficiente de Alfa de Cronbach basado en elementos estandarizados

Tabla 8. Coeficientes Alfa de Cronbach

El significado del segundo factor tiene relación directa con elementos como los ítems 21, 22 y 23 , donde puede inferirse una motivación por la integración de la tecnología en Matemáticas.

Los cuatro parámetros que definen el Factor III (24, 25, 26 y 27) se refieren a aspectos que expresan rechazo al uso de la tecnología pese a reconocer su importancia, lo denominamos como la tecnología no es útil para Matemáticas. Estos cuatro ítems presentan una correlación negativa de elemento totalcorregido.

\begin{tabular}{|c|l|}
\hline Factor & \multicolumn{1}{|c|}{ Descripción de categoría o factor común } \\
\hline I & Utilidad de la tecnología para hacer y aprender matemáticas \\
\hline II & Motivación por la integración de la tecnología en las matemáticas \\
\hline III & No utilidad de la tecnología para el aprendizaje de las matemáticas \\
\hline IV & $\begin{array}{l}\text { Resultado en los estudiantes con respecto al aprendizaje de las matemáticas con el } \\
\text { uso de la tecnología }\end{array}$ \\
\hline
\end{tabular}

Tabla 9. Interpretación de factores relevantes del modelo de 5 factores

El Factor IV integrado por los cuatro parámetros (28, 29,30 y 31) pueden considerarse como el grupo de elementos que tienen como factor común el referir a lo que el estudiante cree o piensa sobre su propio conocimiento, capacidades y habilidades con respecto al uso de la tecnología para las matemáticas.

Interpretamos estos parámetros como referentes a aspectos sobre los resultados del uso de la tecnología en el proceso de enseñanza-aprendizaje de las matemáticas. El grupo de parámetros de este componente también presenta una correlación negativa de elemento total- corregido.

Otro resultado que podemos obtener es que el grupo de parámetros de tres de las sub escalas (Tablas 2, 4 y 5) se dividieron entre dos de los cuatro factores relevantes del modelo. Los parámetros de las dos subescalas restantes (tablas 3 y 6) se encuentran en un solo factor.

\section{Discusión}

Según Gil, Blanco y Guerrero, 2005 una de las áreas del conocimiento dentro de la que se han analizado de forma sistemática es la motivación hacia las Matemáticas y la incorporación de la tecnología para el logro del aprendizaje, teniendo en cuenta el factor motivacional como aspecto determinante en el desarrollo de proceso de enseñanza-aprendizaje por lo que se ha convertido en un desafío según (Cretchley y Harman, 2001) : se incrementa el desafío cuando se añaden factores adicionales, como incorporar la tecnología en las aulas . 
En la presente investigación se observó que un alto porcentaje de estudiantes indicaron que les gustaban las matemáticas con tecnología. Este aspecto demostró que los estudiantes que manifestaron no gustarle las matemáticas con el uso de la tecnología reflejaron falta de motivación y gusto por la asignatura. Investigadores como McClelland (2000), mencionan que la motivación incide notablemente en todas las acciones que el hombre pueda realizar. En este sentido, el rendimiento académico (o desempeño escolar) se puede condicionar en gran medida al componente motivacional. Esto indica que es necesario desarrollar estrategias motivacionales prácticas, que logren un aprendizaje y un gusto por las matemáticas con el uso de la tecnología.

Según (Adell, 2014) los profesores más imaginativos y comprometidos ya han descubierto su potencial y han desarrollado estrategias didácticas bien fundamentadas pedagógicamente para el uso de las Tecnologías de la Información y la Comunicación en el aula y a través de éstas desarrollar de manera eficiente el enfoque comunicativo. Ante esto, es importante mencionar las competencias profesionales del profesor de matemáticas. Poblete Díaz (2003) sostiene que la habilidad adquirida efectiva y eficientemente al ejecutar el acto de enseñar matemáticas.

Entre las competencias generales se contemplan competencias generales y especializadas como habilidad para aplicar conocimientos disciplinarios; habilidad para innovar, indagar y crear en el proceso de enseñanza-aprendizaje de la matemática; capacidad para propiciar un ambiente favorable para el aprendizaje de la matemática; capacidad para lograr una adaptación, actualización y una proyección como profesor de matemáticas (Poblete y Dìaz, 2003).

Respecto a las competencias especializadas del profesor de matemática se tiene por ejemplo: la capacidad para asumir nuevas exigencias curriculares, metodológicas y tecnológicas; destreza para planificar acciones didácticas en matemáticas; capacidad para utilizar diversas estrategias de enseñanza; pericia para comprender, identificar y aplicar teorías de aprendizaje en matemática; capacidad de razonamiento para favorecer el aprendizaje de la resolución de problemas en matemática, por investigación y métodos activos; práctica para seguir, desarrollar y exponer un razonamiento matemático; experiencia para exponer ideas matemáticas; maestría para conectar áreas de desarrollo de la matemática y su relación con otras disciplinas; capacidad para utilizar formas actualizadas en evaluación y esto es un aspecto muy decisivo en el proceso de enseñanza aprendizaje de las Matemáticas utilizando la tecnología que ha influido en la motivación de los estudiantes.
Del mismo modo, las competencias se asocian a marcos de contextos de capacidades del profesor de matemáticas, constituidos por objetos tanto de contenido matemático como didáctico, transversal y evolutivo, que el profesor coloca en juego en su accionar en aula para el logro de la motivación de los estudiantes por el uso de la tecnología en las clases de Matemáticas.

El profesor que pretende enseñar utilizando las Tecnologías de la Información y la Comunicación tiene un rol muy distinto al de un profesor tradicional. Al igual que los estudiantes, debe contar con habilidades tecnológicas mínimas, además de ser experto en el contenido de la materia. Debe ser más consciente de las necesidades del estudiante sin perder los objetivos del programa del curso. Dentro de esas habilidades tenemos, fundamentalmente, que el profesor debe ser motivador; debe ayudar al estudiante a encontrar la coherencia de las actividades que realiza en clase con el mundo real. El profesor debe influir en el estudiante para que este se comprometa, participe y persista en el logro de sus objetivos. (Carneiro, 2019)

Los profesores deben tener en cuenta que su función principal es enseñar a aprender, por tanto, debemos estar capacitados para utilizar la tecnología en el logro del aprendizaje. Así, debemos conocer las técnicas y las herramientas que sean válidas para su aplicación en clase y para la comunicación fuera de la universidad mediante el uso de medios electrónicos. Además, el rol del profesor en la sociedad del conocimiento debe ser el de garante y medio para llevar el conocimiento de las Tecnologías de la Información y la Comunicación a sus aulas.

Para (Pablos, 2012) la formación del profesorado en la era de la tecnología educativa es de gran importancia. Los profesores deben tener una formación específica, tanto en el uso técnico como pedagógico de estos modernos medios.

Gracias al acceso a las tecnologías de las comunicaciones los profesores en la facultad de Ciencias Naturales y de la Agricultura de la Universidad Estatal del Sur de Manabí, podemos disponer en nuestros salones de clases de diversos recursos que nos permiten diversificar el aprendizaje ya sea presencial como virtual facilitando la comprensión de contenidos que explicándolos de una forma teórica sería muy complejo lograr la motivación de los estudiantes por el aprendizaje con el uso de la tecnología. Hoy en día la información en las nuevas tecnologías va revolucionando de manera vertiginosa y los estudiantes a la par, sin embargo los docentes se van quedando rezagados en el manejo de los diversos recursos disponibles. 
El reto que la nueva sociedad plantea a los profesores no se reduce a una actualización científico-didáctica sobre una serie de contenidos de la educación formal, ni se limita a los espacios escolares, sino que ha de tomar parte en la educación no formal de sus estudiantes, potenciando las interrelaciones entre la universidad y sociedad. El concepto de profesor ha tenido con las Tecnologías de la Información y la Comunicación un cambio profundo, por tanto, pasa de ser mero transmisor de conocimientos a guía. El estudiante también cambia, y pasa de ser un sujeto pasivo a adoptar un papel activo.

De manera general, los cuatro factores relevantes que han surgido del análisis factorial muestran la congruencia del cuestionario con la estructura establecida, que trataba de evaluar los cuatro factores iniciales: utilidad de la tecnología en las matemáticas, motivación de los estudiantes por la implementación de la tecnología en las matemáticas, no utilidad de la tecnología en las matemáticas, y resultados de los estudiantes con respecto a su aprendizaje de las matemáticas con el uso de la tecnología. El diseño y validación de la escala elaborada da como resultado un cuestionario válido para medir las actitudes de los estudiantes hacia el uso de la tecnología en el aprendizaje de las matemáticas. Como podemos observar en la Tabla 6, el primer factor explica un $30 \%$ de la varianza y este porcentaje proporciona una evidencia de validez del cuestionario.

Aunque no existe un acuerdo sobre el porcentaje de varianza mínima que debe explicar el primer factor, se sabe, como puede también observarse, que debe ser claramente superior a los factores restantes.

\section{Conclusiones}

El análisis de fiabilidad de la escala construida permite concluir que el test de motivación elaborado es confiable para determinar la motivación de los estudiantes hacia el uso de la tecnología en el aprendizaje de las matemáticas. Este análisis además de asegurar una consistencia interna, permitió identificar los elementos problemáticos que básicamente recaen en los parámetros 2, 24, 25, 26 y 27 del cuestionario situados en el factor residual.

El diseño y validación de la escala elaborada da como resultado un cuestionario válido para medir las actitudes de los estudiantes hacia el uso de la tecnología en el aprendizaje de las matemáticas. Como podemos observar en la Tabla 6, el primer factor explica un $30 \%$ de la varianza y este porcentaje proporciona una evidencia de validez del cuestionario.

\section{Referencias}

Adell, J. (2014). Internet en el aula. Obtenido de http://www.cyta.com.ar/alearn/wq/wq_archiv os/AdellWQ.pdf.

Carneiro, R. (2019). Los desafios de las TIC para el cambio educativo. España: Fundación Santillana.

Cretchley, P. (2007). Does computer confidence relate to levels of achievement in ICTEnriched learning models? Education and Information Technologies, 12(1), 29-39.

Cretchley, P. \& Harman, C. (2001). Balancing the scales of confidence: Computers in early undergraduate mathematics learning. USQ ePrints, Quaestiones Mathematicae, 17-25.

Fogarty, G., Cretchley, P., Harman, C., Ellerton, N., \& Konki, N. (2001). Validation of a questionnaire to measure mathematics confidence, computer confidence, and attitudes to the use of technology for learning mathematics. Attitudes to Technology in Mathematics Learning Questionnaire.

Galbraith, P. \& Haines, C. (1998). Disentangling the nexus: Attitudes to mathematics and technology in a computer learning environment. Educational Studies in Mathematics, 36(3), 275-290.

Galbraith, P. \& Haines, C. (2000). Mathematicscomputing Attitudes Scales. Monographs in Continuing Education. London: City University London.

Gil, N., Blanco, J. \& Guerrero, E. (2005). El dominio afectivo en el aprendizaje de las Matemáticas. Una revisión de sus descriptores básicos. Revista Iberoamericana de Educación Matemática, 2, 15-32.

Gómez-Chacón, I. \& Haines, C. (2008). Students' attitudes to mathematics and technology. Comparative study between the United Kingdom and Spain. Presentado en ICME-11, 11 th International Congress on Mathematical Education. Monterrey: México.

Mcclelland, M. (2000). Children at risk for early academic problems: The role of learning related social skills. Early Childhood Research Quaterly, 15 (3), 307-329.

Nguyen, D. \& Kulm, G. (2005). Using Web-based practice to enhance Mathematics learning and achievement. Journal of Interactive Online Learning, 3(3). 
Pablos, P. J. (2012). Tecnología Educativa: La formación del profesorado en la era de Internet. . Malaga, España: Ijibe.

Poblete, A., Díaz, V. (2003). Competencias profesionales del profesor de matemáticas numéricas. Revista de Didáctica de las Matemáticas 53: 3-13.

Pierce, R., Stacey, K. \& Barkatsas, A. (2007). A scale for monitoring students' attitudes to learning mathematics with technology. Computers \& Education, 48(2), 285-300.

Ursini, S., Sánchez, G. \& Orendain, M. (2004). Validación y confiabilidad de una escala de actitudes hacia las matemáticas y hacia las matemáticas enseñadas con computadora. Educación Matemática, 16(3), 59-78. 